\title{
The Effect of Emotion on Associative Memory: Anger Versus Fear Melissa Adler*, Human Physiology
}

\begin{abstract}
Studies show that emotion enhances memory for individual items but weakens memory for associations between items (Bisby \& Burgess, 2014). One explanation for this associative memory impairment is that emotional stimuli capture attention, causing enhanced encoding of the emotional item but reduced encoding of the surrounding environment (Schupp, Junghöfer, Weike, \& Hamm, 2003). This explanation generates the prediction that emotional information always impairs associative memory. Alternatively, it may be that emotion orients attention towards threats in the environment, suggesting that emotions' effects on associative memory may differ depending on where they indicate a threat may be coming from (Öhman, Flykt, \& Esteves, 2001). For example, seeing an angry face constitutes a direct threat. The angry face itself potentially captures attention and thereby reduces memory for its associated information. In contrast, seeing a fearful face indicates a threat elsewhere in the environment. Therefore, the fearful face may redirect attention towards the surroundings and thus enhance encoding of the associated information. To adjudicate between these hypotheses, subjects studied sets of three images, consisting of two objects and a face with either a neutral, angry, or fearful expression. Subjects were later tested on their memory for the associations between the three items. Supporting the first hypothesis, memory for both angry and fearful associations was worse than memory for neutral associations. Contrary to the second hypothesis, there were no differences in memory for angry versus fearful associations. Thus, emotional information itself seems to capture attention, weakening memory for related information.
\end{abstract}

\section{INTRODUCTION}

\subsection{DEFINING MEMORY}

Memory is involved in many aspects of our everyday lives, from events as simple as remembering to put a foot on the brake when starting a car, to events as complicated as

*Melissa Adler is a 4th year undergraduate student in the Clark Honors College at the University of Oregon. She is a pre-medicine, human physiology major and chemistry minor. She is planning on attending medical school after graduation to become a cardiothoracic surgeon. This research was completed for her honors college/departmental honors thesis, which she defended during the winter of 2020, and passed with distinction. Please direct correspondence to madler@uoregon.edu. 
remembering the steps to an intricate heart surgery. We constantly rely on our memories to inform our thoughts, actions, and relationships, and therefore often trust them as an absolute truth; however, our memory is not always reliable. Emotion affects memory in various ways and can decrease the accuracy of certain types of memory, impacting our day-to-day lives.

There are many forms of memory, with only some types of memory- declarative memoriesbeing accessible to conscious report. Two declarative memory functions have been especially highlighted in the context of emotion influence: item memory and associative memory. Item memory refers to the ability to remember specific, individual items. For example, one could have memories of a specific dog one passed on the street walking home from work every day. Another form of memory is associative memory, which involves the ability to learn and remember the relationships between two or more items. An example would be if the same person not only remembered the specific dog, but remembered it with contextual details such as what its collar looks like, who its owner is, etc.

The two types of memory, associative memory and item memory, are believed to be supported by different brain regions. Associative memory is a distinct form of memory due to its heavy reliance on the hippocampus. The hippocampus is a region of the brain that assists in the formation, organization, and storage of memories, as well as connecting them to certain sensations and emotions; it plays a large role in remembering relationships between multiple pieces of information (Cohen \& Eichenbaum, 1993; O'Keefe \& Nadel, 1978). In contrast, item memory can be supported by regions other than the hippocampus, including the perirhinal cortex as part of the cortical medial temporal lobe (Stark, Bayley, \& Squire, 2002). Due to the different mechanisms underlying item memory versus associative memory, the two may be influenced by contextual factors in distinct ways.

\subsection{EMOTIONAL EFFECTS}

Not all events are remembered, and some are remembered more strongly than others. One factor known to influence memory is emotion. The amygdala plays an integral role in our emotional responses to events. It is located near and often interacts with the hippocampus, affecting the processes of memory formation. It helps determine what and where memories are stored and allows for appropriate responses to dangerous situations (Dolcos, LaBar, \& Cabeza, 2004). The amygdala modulates emotionally influenced memories and works with the hippocampus and other regions of the brain involved in memory to determine what individual elements of the memory are consolidated and stored (Adolphs, Cahill, Schul, \& Babinsky, 1997; Phelps, 2004). It is likely that emotional events are coded differently than neutral events, such as the amygdala being responsible for encoding as opposed to the hippocampus. This distinction could explain the qualitatively different memories resulting from events involving different states of emotional arousal.

For a long time, it was believed that emotion enhanced memory, but the studies from which that conclusion was based mostly focused on memory for individual emotional items, such as angry faces (Bradley, Greenwald, Petry, \& Lang, 1992; Brown \& Kulik, 1977; Chiu, Dolcos, 
Gonsalves, \& Cohen, 2013). More recent work shows that associative memory for emotionally related information may be impaired compared to those for neutral information. For example, following the events of 9/11, people remembered the first plane striking the first tower, yet forgot where it hit, how long it took for the tower to collapse, and even where they saw/heard about the event-the majority of people incorrectly claimed that they had witnessed the first strike on video, though it was not televised (Pezdek, 2003). This is likely due to the way that the amygdala affects the hippocampus, weakening and impairing the encoding process for associative memories; this can be seen by low hippocampal activity in the presence of negative emotion, corresponding to reduced associative memory (Bisby \& Burgess, 2017). However, increased activation of the amygdala in the presence of negative emotion improves specific item memory. Because of this, a person may remember individual elements of an event yet be unable to form a relationship between multiple elements.

Emotional stimuli often capture attention, which causes surrounding information to be ignored, and, as a result, memory increases for the emotional stimuli while it decreases for the contextual details (Schupp, Junghöfer, Weike, \& Hamm, 2003). Attention may also be captured by the presence of potential threats. Past research indicates that attention is driven by physiological arousal via activation of the amygdala; threats evoke emotional arousal that leads to activation of the amygdala, which can then capture attention to the detriment of nonthreatening information in the environment (Öhman, Flykt, \& Esteves, 2001). Furthermore, item memory involves focusing on a single, independent item, leading to strong memory of it, while associative memory places a different demand on attention. With associative memory, attention is required to be spread across multiple items to ensure encoding of their relationships.

\subsection{RESEARCH QUESTIONS AND HYPOTHESES}

Studies show that specific item memory (e.g., remembering a face or object) is typically enhanced in the presence of emotion; however, associative memory is weakened in the presence of emotional content (Bisby \& Burgess, 2017). Therefore, if an angry face is seen paired with an object, a person is more likely to remember the face but less likely to remember the associated object. One explanation for this is that emotional stimuli capture a person's attention, which causes the surrounding environment to be ignored (Schupp et al., 2003). Since attention is what drives encoding, this would lead to a lack of encoding of the surrounding environment. In this case, all emotional stimuli should elicit a deficit in the formation of associative memories. Another explanation is that a person's attention is oriented towards threats in the environment, which may lead to differences in associative memory based on the type of emotional content depicted (Öhman et al., 2001). Angry faces might lead people to forget the surroundings because the threat is the angry face itself. However, if the face is instead fearful, people may direct their attention towards the items in the surrounding environment to locate the source of the threat.

This study compares these two explanations of how emotion affects associative memory, specifically testing whether perceptions of anger and fear have different effects on memory for associated information. We tested two competing hypotheses: 1) that emotional faces, both angry and fearful ones, would inhibit associative memory compared to neutral faces and 2) that fearful 
faces would allow subjects to form better associative memories than angry faces (Figure 1). This is because, with the fearful stimuli, subjects' attention would likely transfer from such stimuli to the objects, the potential sources of the threat, while the angry stimuli would act as the sources of the threat themselves and would hold the subjects' attention. These results provide new evidence about the cognitive mechanisms underlying emotional effects on memory. Adjudicating between these two hypotheses not only provides a novel understanding of the basic mechanisms of memory, but also has implications for the reliability of eyewitness testimony and for the types of cognitive impairments that could result from traumatic experiences.

Hypothesis 1:
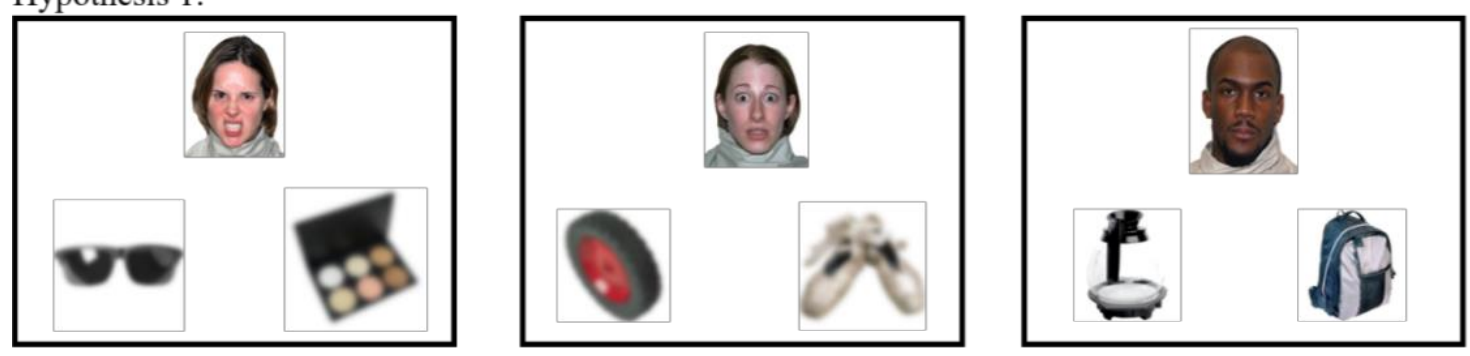

Hypothesis 2:
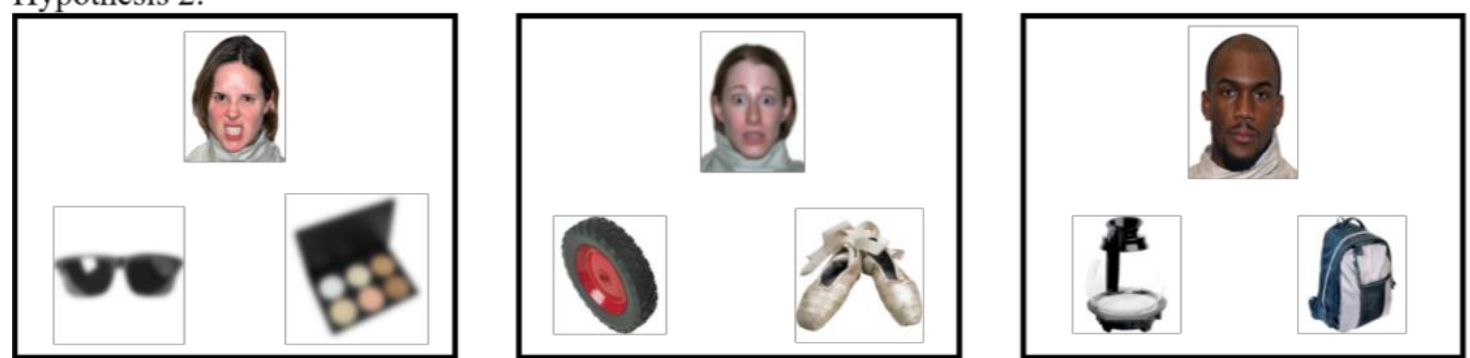

Figure 1: Potential hypotheses for how emotion affects associative memory. Hypothesis 1: emotion itself captures attention-both angry and fearful faces will disrupt associative memory. Hypothesis 2: emotion signals where to direct attention- angry faces (threats themselves) disrupt associative memory while fearful faces (threats in environment) do not.

\section{METHODS}

\subsection{SUBJECTS}

Forty University of Oregon undergraduate students participated for course credit (age range = $18-27$, mean age $=19.85$, standard deviation $=2.48 ; 30$ females and 10 males). All subjects provided informed consent in accordance with regulations set by the University of Oregon and the Institutional Review Board prior to participation and were given a debriefing following their study completion.

\subsection{STIMULI}

Face stimuli were taken from a pool of 125 face images consisting of 18 male-appearing and 18 female-appearing faces. Face stimuli were taken from the database NimStim (Tottenham et al., 2009), which is composed of images from actors and actresses in New York University's theater 
department. Each face was photographed with three emotional expressions: neutral, angry, and fearful, though each subject was randomly assigned only one of the three facial expressions provided by the same actor. There were an equal number of male-appearing and female-appearing faces in each of the three emotion conditions.

Each face was paired with two objects to form an associative triad, with the face at the top of the screen and the two objects at the bottom. There were 108 object stimuli taken from the internet that were all categorized as neutral by experimenter judgment. The pairings between faces and objects were random for each subject so they viewed different triads, though every subject viewed all stimuli over the course of the experiment.

Not all objects were presented in triads; some objects were used individually in a different experimental phase to represent "new" items, as in previously unstudied, as a means of assessing subjects' recognition. The 36 object stimuli that remained after creating the face-object triads were used as "new" items during the recognition phase.

\subsection{EXPERIMENTAL DESIGN}

The study was entirely computer-based and was coded using MATLAB Psychtoolbox software (https://www.mathworks.com/products/matlab.html). There were three experimental phases: study, recognition, and testing. The experiment lasted approximately one hour for each subject. Instructions for each of the phases were given to the subjects before the tasks began. Subjects completed practice rounds for the study and testing phases, consisting of the same 14 object stimuli and the same seven face stimuli taken from the entire experimental pool of stimuli, for all subjects. The practice face and object stimuli were not used in the actual experiment. The directions provided and the practice rounds completed did not inform subjects of the emotion manipulation, so subjects were unaware of the specific focus of the study in order to maintain unbiased results.

\subsubsection{STUDY PHASE}

For each study trial, subjects saw a single triad and were asked to create a mental image or story connecting all three items so they could more easily remember the images' connections in the future (e.g., the angry woman wore sunglasses as she put on her makeup) (Figure 2). Triads were always presented with the face at the top of the screen and the two objects on the bottom. Each triad was present on the screen for six seconds, during which time subjects ranked the quality of their stories on a scale of one to four, one meaning that they were unable to come up with a story to help them remember the relationships between the images, and four meaning that their story was of high quality. Between each triad, a fixation cross was present for two seconds. Thirty-six triads were studied for this phase, 12 of each emotion condition (e.g., of neutral, angry, and fearful facial expressions). 


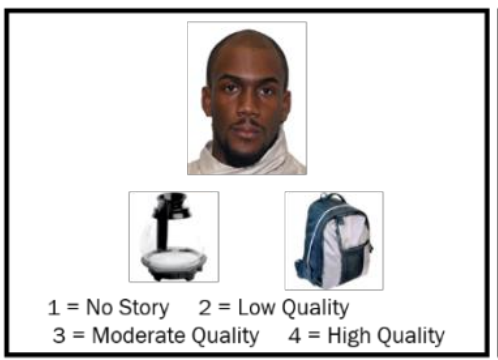

Neutral Triad

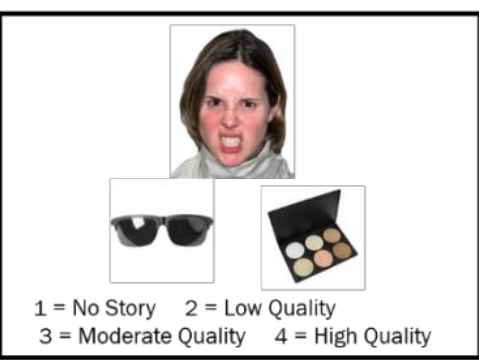

Angry Triad

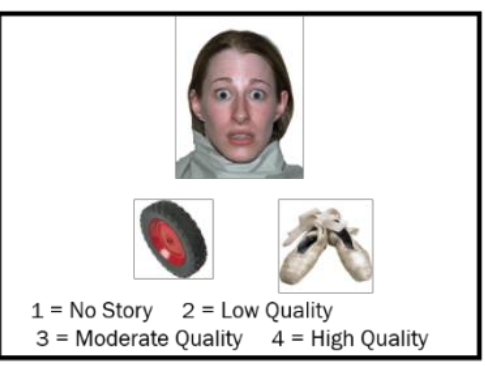

Fearful Triad

Figure 2: Study phase. Each box represents the computer screen during one trial of the study phase. Each trial consisted of one face at the top of the screen with two objects underneath, called triads. Subjects studied these triads, either with a neutral, angry, or fearful face, for six seconds, during which time they came up with stories connecting the three items and ranked the quality of those stories using the rankings provided across the bottom of the screen.

\subsubsection{RECOGNITION PHASE}

Subjects then completed the recognition phase of the experiment, consisting of solely object stimuli (Figure 3). For each round, one object appeared in the middle of the screen and subjects determined if the object was one that they had been shown before, in the previous phase, or if it was one that they had not been shown before. Each image was present on the screen for six seconds, during which time subjects pressed one, indicating that the image was "old", or two, indicating that the image was "new." This round consisted of 108 trials. There were 36 "new" and 72 "old" stimuli for each subject.

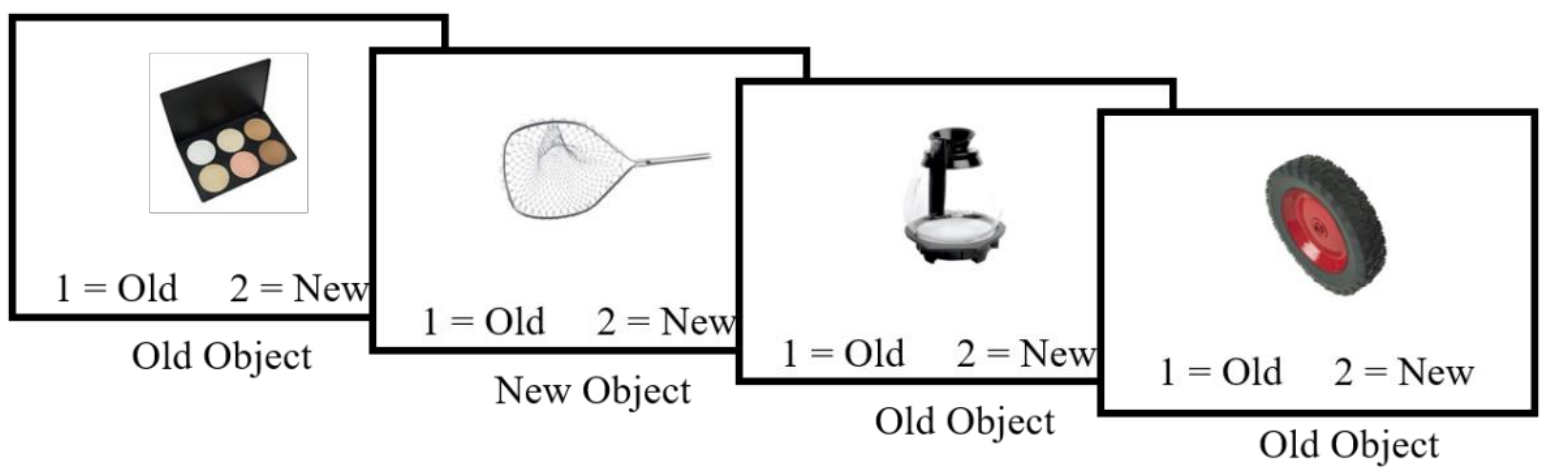

Figure 3: Recognition phase. Each box represents the computer screen during one trial of the recognition phase. Each trial consisted of a single object that remained on the screen for six seconds, during which time subjects determined if the object was "old," being one that they had studied during the prior phase, or if the object was "new," being one that they had not studied during the prior phase.

\subsubsection{TESTING PHASE}

Finally, subjects were tested on their ability to form associative memories between the faces and the objects they studied in the first phase (Figure 4). Seven images were present on the screen, with the image on top acting as the cue image, and the six images across the bottom acting as answer choices. Subjects indicated which one of the six images were originally paired with the cue image by typing the number assigned to their chosen answer choice. 
The six answer choices across the bottom consisted of two images from neutral triads, two images from angry triads, and two images from fearful triads. Including multiple images from each emotion condition allowed us to test how often participants remembered the correct emotion (e.g., that the cue item came from an angry triad) without remembering the specific face or object associated with the cue (e.g., from Figure 4, not remembering whether the fearful face was associated with the fossil or the hour glass). The two stimuli from each emotion condition were arranged in columns so that there were three columns of two stimuli. For example, the two leftmost images might always be from neutral triads, the middle images from fearful triads, and the rightmost from angry triads. While this arrangement would be consistent across the entire testing phase for a given subject, which condition was associated with which column was counterbalanced across subjects.

Each triad was tested in every possible cue-answer choice combination, meaning that every triad was tested 6 different ways: face cue-object 1 answer, face cue-object 2 answer, object 1 cueface answer, object 1 cue-object 2 answer, object 2 cue-face answer, object 2 cue-object 1 answer. For example, if a fearful face was paired with an hourglass and an apple during study, then this triad would be tested under the following six permutations: fearful face as cue - hourglass as correct answer, fearful face as cue - apple as correct answer, hourglass as cue - fearful face as correct answer, hourglass as cue - apple as correct answer, apple as cue - fearful face as correct answer, apple as cue - hourglass as correct answer. Testing all possible permutations allowed for a robust estimation of how well individuals had bound all three items together in memory for each triad.

This phase was self-paced and consisted of 216 trials. All answer choices were images that had been studied prior; there were no "new" images.
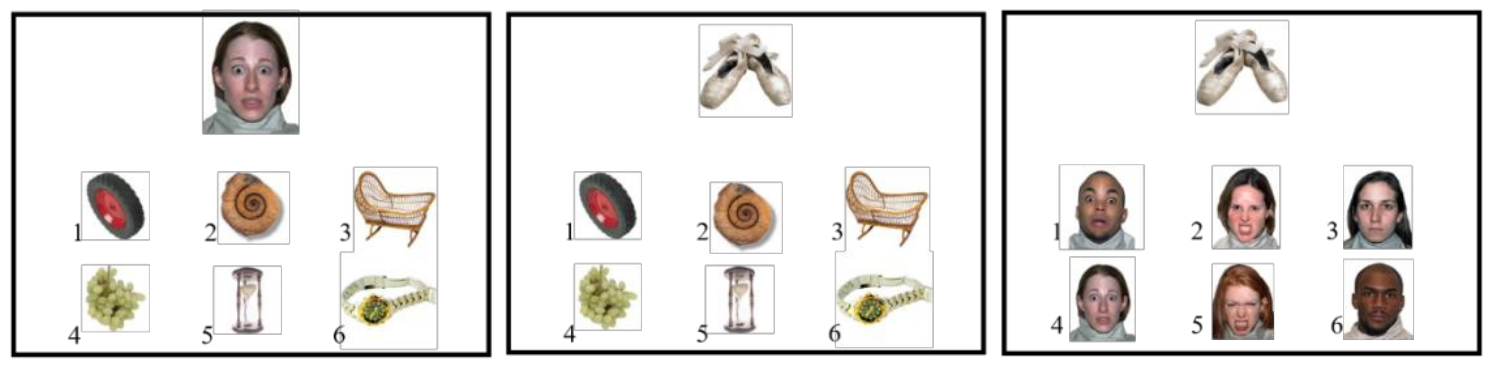

Figure 4: Association test phase. Each box represents the computer screen during one trial of the association test phase. Each trial consisted of one item at the top of the screen, representing the cue item, and six items underneath the cue item, representing the answer choices. Subjects determined which one of the six answer choices were in a triad with the cue item during the study phase. Each triad from the study phase was tested in every possible way. This phase was self-paced.

\subsubsection{QUESTIONING}

After the three phases were completed, subjects were asked two questions to determine if their emotional arousals were affected by the study. The first question asked if the subjects had any particular feelings while they were completing the study phase of the experiment. The second question asked if the study phase made the subjects feel more negative, neutral, or positive overall. The first question was open-ended so as not to influence the subjects' responses, while the second 
question was meant to direct the subjects' responses to specific emotional states. Answers were recorded on each subjects' corresponding line on the sign-in sheet. Notes were also written for any technical issues that came up during the study and for subjects who had any medical conditions or were in a state of mind that may have influenced their results and affected the data.

\section{RESULTS}

\subsection{TRAINING}

In training, our dependent measure of interest was the rating of story quality. For each subject, we computed a mean rating separately for each of the three emotion conditions (Figure 5). We then submitted these mean ratings to a one-way, repeated-measures ANOVA. The effect of emotion condition on training rating was not significant $\left(F_{(2,98)}=2.12, p=0.13\right)$. Though no overall emotion effect was confirmed, we ran exploratory, pairwise comparisons between the fearful triads and the neutral and angry triads. Ratings were numerically higher for faces from fearful triads than faces from angry $(\mathrm{t}(49)=1.70, p=0.10)$ or neutral triads $(\mathrm{t}(49)=1.66, p=0.10)$; however, differences among conditions were not significant.

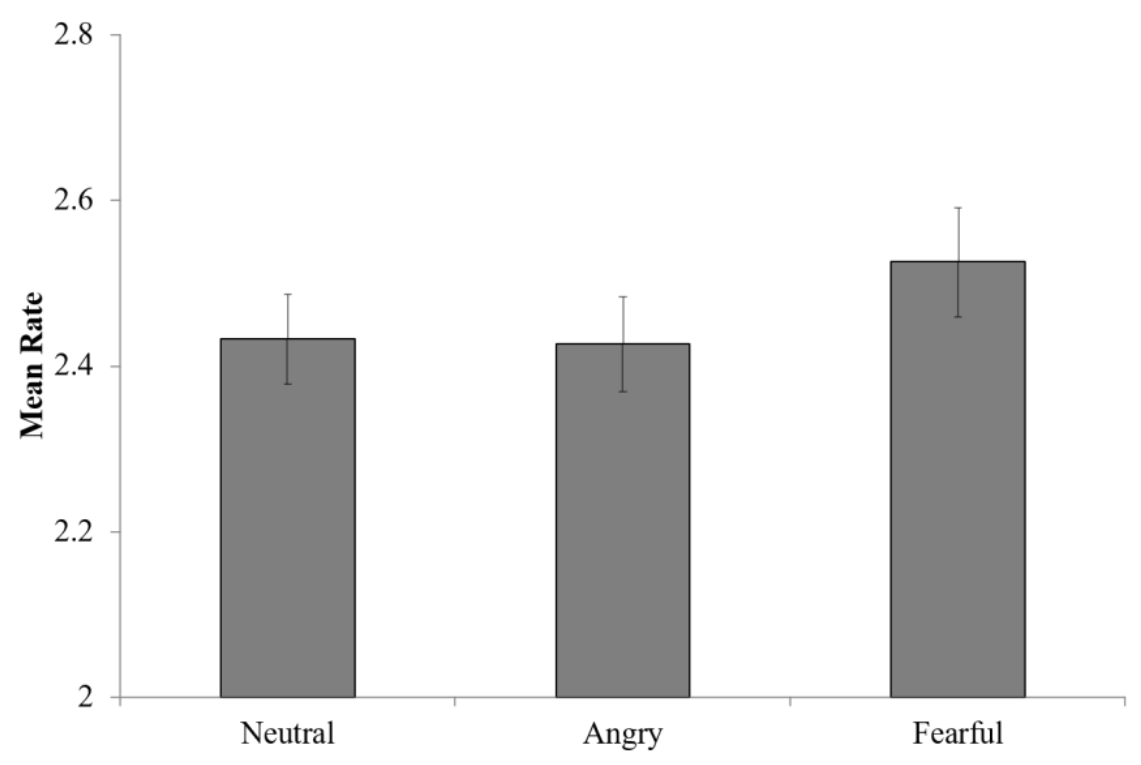

Figure 5: Mean ratings during training. The mean quality of story ratings in each emotion condition (e.g., neutral, angry, and fearful). Error bars represent across-subject standard error of the mean. Although fearful triads were rated higher numerically, differences among conditions were not statistically reliable.

\subsection{RECOGNITION}

It is possible that attention is captured by emotion itself, which may lead to a lack of encoding of the paired objects in angry and fearful triads. Thus, to determine if emotion influenced object recognition, our dependent measure of interest was the mean proportion correct for identifying old objects when they were in fact old (Figure 6). We submitted these values to a one-way, 
repeated measures ANOVA. Data showed no effect of emotion, with subjects performing comparably across all conditions $\left(F_{(2,98)}=0.26, p=0.77\right)$.

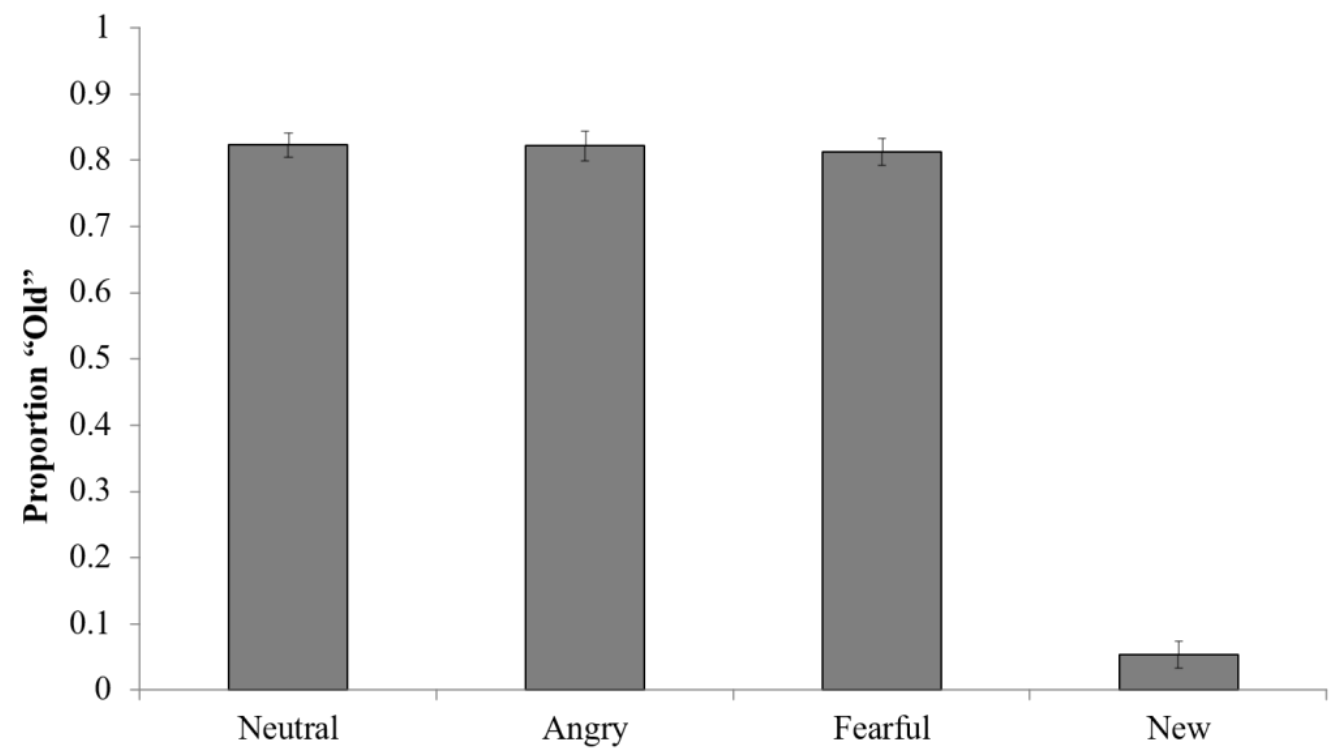

Figure 6: Proportion of "old" responses during recognition. The mean proportion of times subjects responded "old" during recognition for each of the emotion conditions. Error bars represent acrosssubject standard error of the mean. Response accuracy did not differ across emotion conditions.

\subsection{ASSOCIATION TEST}

\subsubsection{PROPORTION CORRECT}

We calculated mean proportion correct to assess associative test performance for each emotion condition, separated by cue-answer choice trial type (Figure 7). A 3 (emotion condition: neutral, angry, fearful) x 3 (cue-answer choice type: face-object, object-object, object-face) repeated measures ANOVA was completed to assess the effects of emotion and test type, along with the interaction between them. Results showed a significant overall effect of emotion $\left(F_{(2,98)}=7.13, p\right.$ $=0.001$ ). Subjects were better at remembering neutral triads (mean $=0.50, \mathrm{SE}=0.18$ ) compared to both angry (mean $=0.45, \mathrm{SE}=0.17 ; \mathrm{t}(49)=3.40, p=0.001)$ and fearful triads $($ mean $=0.47$, $\mathrm{SE}=0.19 ; \mathrm{t}(49)=2.58, p=0.01)$. Although the accuracy for fearful trials was numerically higher than for angry triads, the difference was not statistically significant $(\mathrm{t}(49)=-1.23, p=0.22)$. Results also indicated a significant overall effect of test type $\left(F_{(2,98)}=125.05, p<0.001\right)$. Subjects performed significantly better for the object cue/object answer choices trials than the face cue/object answer choices trials $(p<0.001)$ and the object cue/face answer choices trials $(p<$ o.001). Subjects had significantly greater accuracy when tested with the object cue/face answer choices format than with the face cue/object answer choices format ( $p=0.002)$. The interaction between test type and emotion condition was not significant $\left(F_{(4,196)}=1.18, p=0.321\right)$. Subjects' associative memories were similarly inhibited by negative emotion across all test types. 


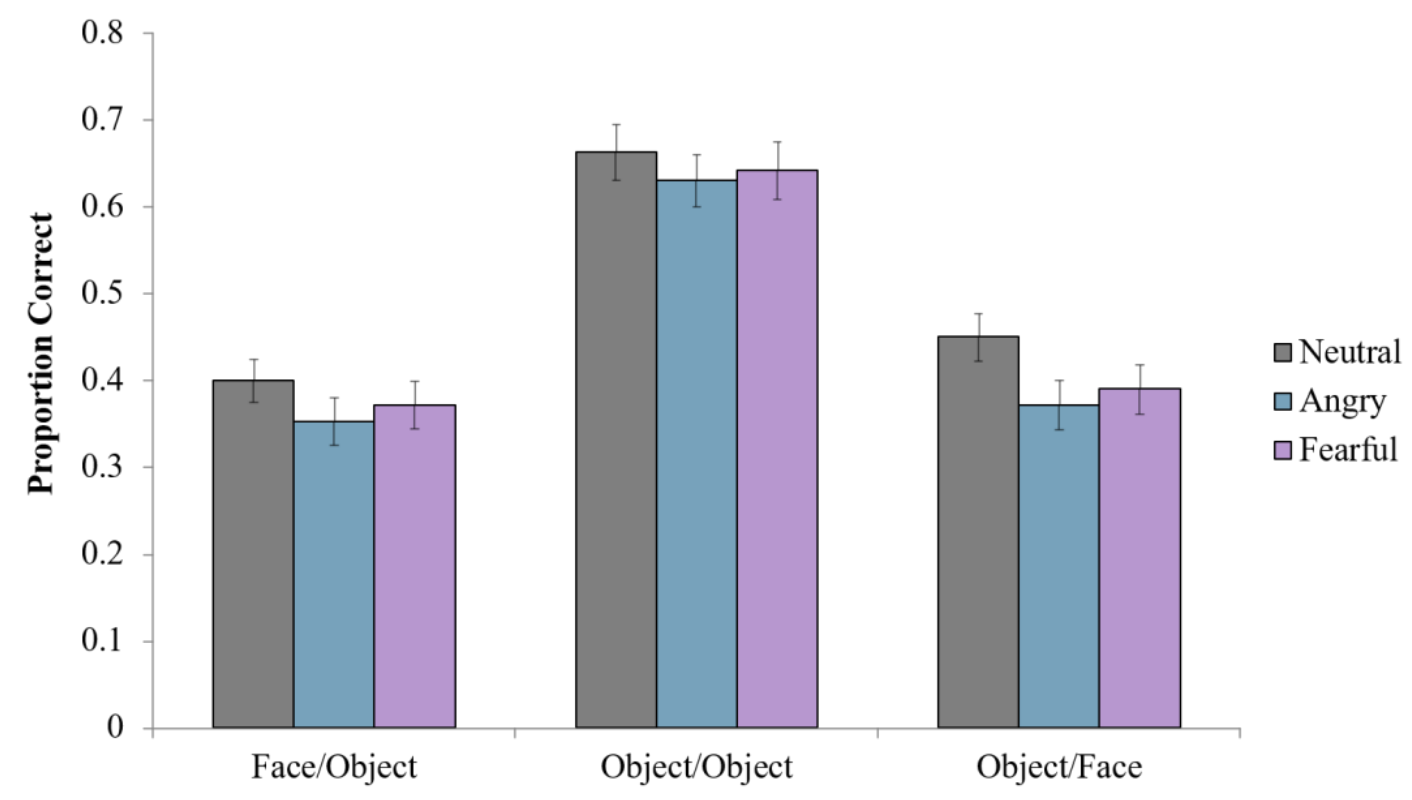

Figure 7: Proportion correct during association test. The effect of emotion on associative memory performance, represented by mean proportion correct, separated by test type. Error bars represent across-subject standard error of the mean. Subjects exhibited higher performance for neutral triads compared to emotional triads, with fearful triad performance being numerically higher than angry

triad performance. Subjects performed highest for the object cue-object answer choice testing condition. No interaction between emotion condition and testing condition were found.

\subsubsection{PROPORTION OF EMOTION-CONSISTENT ERRORS}

One possibility is that if emotion itself captures attention, individuals might remember the emotion itself but not the specific face. To test whether subjects had emotion memory but not memory for specific faces, we measured the proportion of incorrect responses where subjects chose the wrong items but where such items were from triads with the correct emotion conditions (Figure 8). Although subjects showed a numerical tendency to select the wrong item from the correct emotion condition for angry or fearful faces more so than neutral faces, the effect of condition was not significant (one-way, repeated measures ANOVA $\left(F_{(2,98)}=2.08, p=0.13\right)$. 


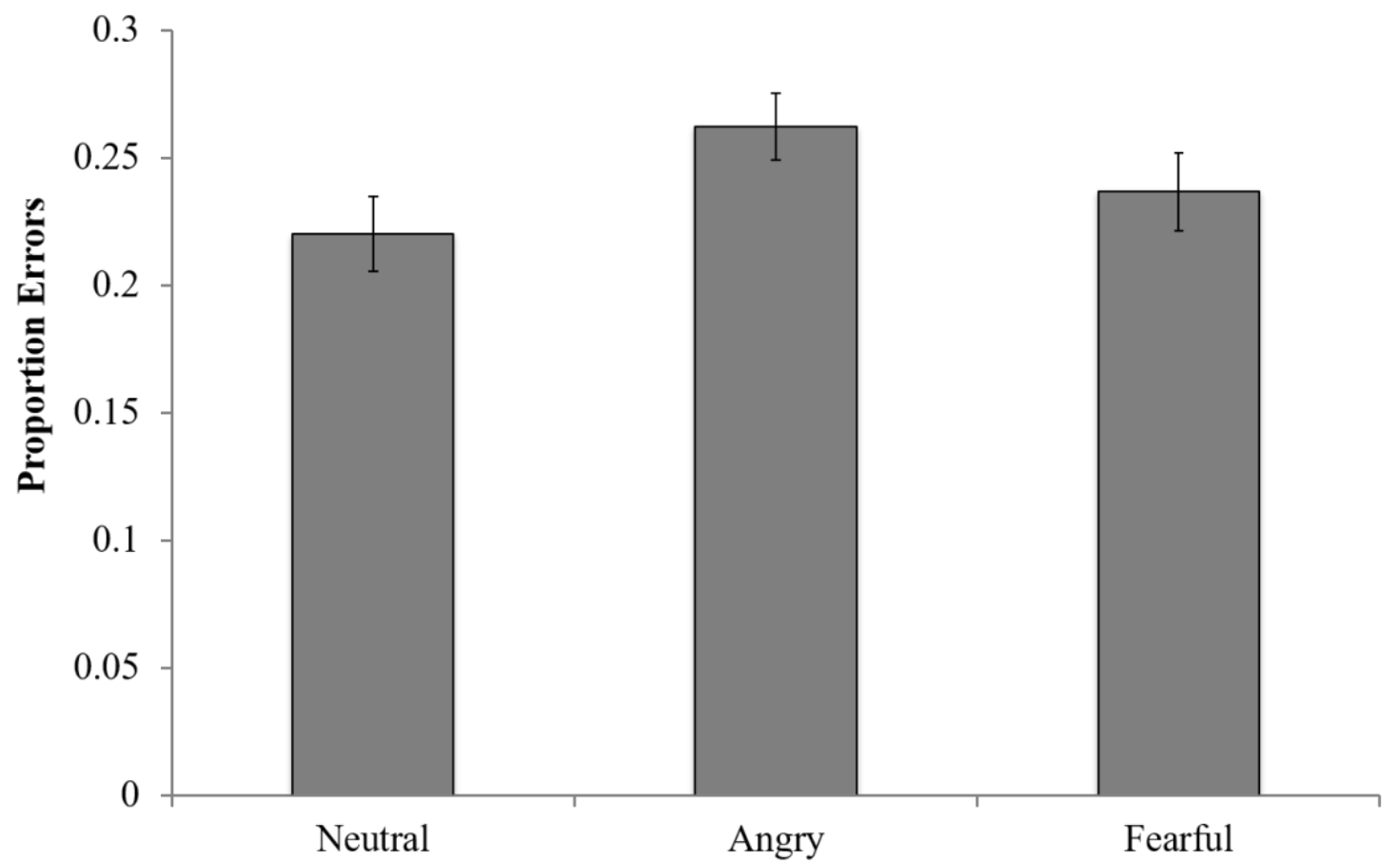

Figure 8: Proportion of emotion-consistent errors during association test. The effect of emotion on subjects' abilities to recognize the correct emotion in instances when they did not identify the correct, specific face. Error bars represent across-subject standard error of the mean. No overall significance of emotion condition was found.

\section{DISCUSSION}

The goal of this study was to better understand how emotion modulates memory. We investigated how the presence of negative emotion affects the formation of associative memories, and if different types of negative emotions cause varying effects. Two competing hypotheses for how negative emotion influences associative memory were tested. The first hypothesis was that the presence of negative emotion, regardless of specific type, would attract attention to the emotional stimulus at the expense of memory for associated information. The second hypothesis was that fear would lead to stronger associative memory formation compared to anger because fear would direct attention to potential threats in the environment. To test these hypotheses, subjects studied three-item pairings consisting of two neutral objects and a face displaying either a neutral, angry, or fearful expression. Subjects were tested on their memory for individual objects as well as associative memories for the three item pairings they studied originally. Although there was no difference in memory for individual items, neutral triads were better remembered. There was no significant difference between the two negative emotions, anger and fear, in terms of subjects' recognition of individual items or associative test performance. Based on this information, our data support the first hypothesis, indicating that negative emotion inhibits associative memory formation independent of specific type. 


\subsection{NEGATIVE EMOTION INHIBITS ASSOCIATIVE MEMORY FORMATION}

Our results support the typical findings of prior research investigating this topic. Many studies have shown that the presence of negative emotion decreases the associative binding of items within their proper context, leading to fragmented associative memories (Bisby \& Burgess, 2014, 2017; Madan, Caplan, Lau, \& Fujiwara, 2012; Mather \& Knight, 2008; Rimmele, Davachi, Petrov, Dougal, \& Phelps, 2011). Although the reason behind this is not entirely understood, it has been theorized that negative emotion disrupts associative binding of peripheral information (Touryan, Marian, \& Shimamura, 2007), or that negative emotion disrupts associative encoding by impairing pattern completion-a series of neural connections that, if triggered, fire one another and lead to memory recall-and therefore memory of the event as a whole (Bisby, Horner, Bush, \& Burgess, 2018). Regardless of the reasoning behind such theories, our data indicate that anger and fear cause similar effects, which leads us to conclude that negative emotion, in general, inhibits associative memory formation.

\subsection{ASSOCIATIVE IMPAIRMENT PRESENT FOR BOTH FEAR AND ANGER}

Though our second hypothesis predicted associative memory differences between angry and fearful triads due to their varying sources of threat, this was not the case for the present research, as both types of emotional triads produced comparable levels of memory impairment. Prior work has shown that selective attention enhances encoding of highly relevant stimuli, with the most emotional stimuli being of the greatest importance and therefore being encoded the strongest (Schupp et al., 2003). Fearful faces themselves might be less relevant compared to the environments around them since the cause of the emotion must be located in the surroundings. Alternatively, attention may also be captured by the presence of threats. Prior research has shown that people automatically turn their attention towards stimuli that pose a threat, a potential evolutionary trait acquired to alert us to danger (Öhman et al., 2001). Based on this information, one. would predict that angry stimuli would capture one's focus and lead to decreased encoding of the surrounding environment, while fearful stimuli would redirect focus towards the surrounding environment and therefore increase encoding of the paired items. Our results instead indicate that the angry and fearful triads did not differentially recruit this selective attention mechanism. This information leads us to believe that it is emotion in general that influences attention, and that anger and fear may not be discriminated in terms of their emotional significance and encoding, leading to the similar associative memory results that our data show. However, it is possible that fearful faces sometimes do direct attention toward the environment, and that our stimuli did not pose any degree of real threat. In future studies, we could use physiological measures of arousal to test for evidence of threat associated with fearful and angry faces, though our data are more consistent with the hypothesis that selective attention is modulated via emotional significance, rather than by threat.

An alternative hypothesis to that of the selective attention mechanism is that negative emotion causes anxiety, which then causes poor associative encoding. Studies indicate that the feeling of anxiety in the presence of an angry or threatening face inhibits processing efficiency, which leads to decreased encoding and poorer associative memory (Derakshan \& Koster, 2010). Increased 
anxiety may also be present in the fear condition; rather than viewing the face itself as threatening, the threat stems from the environment, causing a similar feeling of anxiousness, though from a different source. Our data show similar associative memory effects from angry and fearful triads, indicating that emotional arousal leads to a poor state for encoding. Thus, our data could be the result of anxiety as a response to the presence of threat.

\subsection{DIFFERENTIAL EFFECTS OF ASSOCIATIVE AND ITEM MEMORY}

Our results also support prior work showing that negative emotion affects associative memory differently from item memory. A long line of research has shown that portions of the medial temporal lobe (MTL) are critical for forming memories (Stark et al., 2002). One subregion, the hippocampus, is especially important for forming associative memories, while others support memory for individual items (Bisby \& Burgess, 2014). Specifically, our data show associative memory deficits without differences in item recognition between conditions. This provides further support for the idea that the two types of memory, associative and specific item, are supported by different mechanisms. Recognition relies upon MTL structures other than the hippocampus, and retrieval of a single item does not necessarily rely on contextual details or item associations and mainly requires extra-hippocampal support (Montaldi \& Mayes, 2010), while associative memory relies heavily upon those factors and requires hippocampal involvement (O’Keefe \& Nadel, 1978). This may explain why we found differences in how negative emotion affected associative memory versus item memory.

\subsection{AMYGDALA INVOLVEMENT}

Located near the hippocampus, the amygdala, which is involved in emotion regulation, has been shown to play a role in memory. Via connectivity with the MTL, the amygdala can enhance encoding of relevant information. Some work suggests that amygdala activation indexes fear (Davis, 1992; LeDoux, 2007), whereas later work shows that it responds to salient stimuli more broadly (Cunningham \& Brosch, 2012). Our behavioral finding is more in line with this latter account of amygdala processing and shows that negative emotion affects behavior similarly, without a special role for fear in emotional memory.

\subsection{AROUSAL STATE INFLUENCES ENCODING AND RETRIEVAL}

Finally, the associative memory deficit was present throughout the various ways the information was tested (e.g., the cue vs. answer choice layouts). When subjects were tested on object-object associations (e.g., there was no face and therefore no emotion present during the test trial), the deficit for negative emotion remained. The presence of emotion increases arousal during memory encoding, leading to the release of neurotransmitters that modulate memory strength (Cahill \& McGaugh, 1996). When retrieving an emotional event, the amygdala and related brain structures may become re-activated, causing one to be in a similar state of arousal as to when the memory was formed. If this re-activation is too weak or fails to occur, retrieval is suboptimal and memory accuracy decreases (Buchanan, 2007). One potential explanation for the results in our study is that it was not the emotional item capturing subjects' attention during the 
test, but the subjects' differing states of arousal during encoding and retrieval that led to our result; the strength of the negative arousal that was present during encoding was likely absent or too weak during retrieval, hindering subjects' abilities to recall the item pairings.

\subsection{LIMITATIONS}

A primary concern arisen from our behavioral results was that we did not test item memory for the individual faces, so it is possible that the different associative memory effects between the emotional and neutral conditions was reliant upon how well the faces were remembered. Furthermore, we had no measure of amygdala activation or galvanic skin response to ascertain whether subjects' emotional arousal levels were influenced by the faces they were shown. If participants were not emotionally aroused, the differential emotion effect may be due to merely remembering the facial expressions themselves, the emotional facial expressions having more variation amongst them and thus potentially proving more challenging to recall accurately.

\section{CONCLUSIONS}

The present research has important implications for situations where the inference of emotion on memory accuracy is problematic, such as in the criminal justice system. Eyewitness testimonies depend upon witnesses' abilities to remember not only individual elements of events (e.g., having seen a specific person before), but remember how the elements of the event are associated with one another (e.g., having seen the person and the gun together). The events involved are usually emotionally charged, but past studies on the influence of emotion on memory have focused primarily on how people remember individual elements rather than associations between multiple elements. When witnesses provide their testimonies, it is important for them to remember not only the faces of the perpetrators, but the clothes the perpetrators were wearing along with other associated details that may help identify them. It has been shown that eyewitness testimonies are often unreliable, as emotional stress leads to the weakening of memories; negative emotion present during an event or period of recall significantly hinders one's ability to remember details about the event (Kassin, Ellsworth, \& Smith, 1989). This is believed to be due to the idea that there is a decreased capacity to process information in a state of heightened emotional arousal (Kaplan, Van Damme, Levine, \& Loftus, 2016; Van Der Kolk, 1998). Therefore, these testimonies are filled with gaps that the brain attempts to fill in with information based on other surrounding pieces of evidence, as well as biases due to personal experiences (Loftus \& Pickrell, 1995). Results from the present work demonstrate challenges involving eyewitness testimonies. Data show that emotion in general, both anger and fear, disrupt associative memory processes; thus the validity of eyewitness testimonies falls further into question. Data also reveal that anger and fear do not have different effects on associative memory, which implies that witness testimonies involving different types of emotional events provide similar amounts of unreliability.

Furthermore, people who experience trauma with some regularity (e.g., victims of abuse) may have cognitive impairments in addition to emotional trauma (Power, Philippot, \& Hess, 2010). Our data suggest that such impairments may be possible not only when an individual experiences direct threats, but when in the presence of any negative emotion like witnessing someone else's 
fear. Importantly, results from this study, along with future variations of it, could provide potential clinical applications involving victims of traumatic experiences; if we understand the mechanisms of how negative emotion influences associative memory, we may discover methods of counteracting the impairment via various memory-improving techniques. This could potentially lead to prevention and/or improvement of cognitive impairment resulting from trauma, improved memory accuracy for emotional events, and increased reliability of eyewitness testimonies.

Although current data indicate that fearful and angry stimuli cause similar associative memory effects, it is possible that the fearful faces did not elicit enough threat perception to orient subjects towards the environment. Future research would likely involve the use of stimuli of higher emotional valence to achieve the desired direction of attention. It would also be beneficial to use Galvanic Skin Response (GSR) to monitor subjects' states of emotional arousal via changes in their sweat gland activity. With GSR we could ensure that subjects do not merely view emotional stimuli but become emotionally aroused themselves. With their electrodermal activity being measured, we could also test whether fear and anger cause varying states of arousal. Furthermore, we could utilize Functional Magnetic Resonance Imaging (fMRI) to measure subjects' amygdala activation; thus, we could determine if anger and fear cause varying levels of amygdala activation and monitor the relationship between associative memory formation and amygdala activation.

The purpose of this experiment was to study two different types of negative emotion and their potential differing effects on associative memory. Overall, our data found the same effect for both emotion conditions, indicating emotional content captures attention, reducing resources available to encode related information. Our results also indicate that the source of threat, whether it be the stimuli themselves if angry or the surrounding environment if fearful, does not influence the strength of associative memories. With this contribution, we further support the idea that negative emotion inhibits associative memory formation and are brought closer to understanding how the effect is modulated.

\section{ACKNOWLEDGEMENTS}

I would like to thank Dr. Dasa Zeithamova for providing me the opportunity to delve into the brain and the fascinating complexities of memory. A special debt of gratitude is owed to my amazing mentor, Caitlin Bowman, for her patience and unwavering support throughout this entire process. I am immensely grateful for the other members of the Brain and Memory lab who fostered a supportive and enriching learning environment where I could hone my research skills and develop cherished friendships.

\section{REFERENCES}

Adolphs, R., Cahill, L., Schul, R., \& Babinsky, R. (1997). Impaired declarative memory for emotional material following bilateral amygdala damage in humans. Learning and Memory, 4(3), 291-300. https://doi.org/10.1101/lm.4.3.291 
Bisby, J. A., \& Burgess, N. (2014). Negative affect impairs associative memory but not item memory. Learning and Memory, 21(1), 21-27. https://doi.org/10.1101/1m.032409.113

Bisby, J. A., \& Burgess, N. (2017, October 1). Differential effects of negative emotion on memory for items and associations, and their relationship to intrusive imagery. Current Opinion in Behavioral Sciences, Vol. 17, pp. 124-132. https://doi.org/10.1016/j.cobeha.2017.07.012

Bisby, J. A., Horner, A. J., Bush, D., \& Burgess, N. (2018). Negative emotional content disrupts the coherence of episodic memories. Journal of Experimental Psychology: General, 147(2), 243-256. https://doi.org/10.1037/xgeoooo356

Bradley, M. M., Greenwald, M. K., Petry, M. C., \& Lang, P. J. (1992). Remembering Pictures: Pleasure and Arousal in Memory. Journal of Experimental Psychology: Learning, Memory, and Cognition, 18(2), 379-390. https://doi.org/10.1037/0278-7393.18.2.379

Brown, R., \& Kulik, J. (1977). Flashbulb memories. Cognition, 5(1), 73-99. https://doi.org/10.1016/0010-0277(77)90018-X

Buchanan, T. W. (2007). Retrieval of Emotional Memories. Psychological Bulletin, 133(5), 761779. https://doi.org/10.1037/0033-2909.133.5.761

Cahill, L., \& McGaugh, J. L. (1996). Modulation of memory storage. Current Opinion in Neurobiology, 6(2), 237-242. https://doi.org/10.1016/So959-4388(96)80078-X

Chiu, Y. C., Dolcos, F., Gonsalves, B. D., \& Cohen, N. J. (2013). On opposing effects of emotion on contextual or relational memory. Frontiers in Psychology, Vol. 4. https://doi.org/10.3389/fpsyg.2013.00103

Cohen, N. J., \& Eichenbaum, H. (1993). Memory, amnesia, and the hippocampal system. MIT Press.

Cunningham, W. A., \& Brosch, T. (2012). Motivational salience: Amygdala tuning from traits, needs, values, and goals. Current Directions in Psychological Science, 21(1), 54-59. https://doi.org/10.1177/0963721411430832

Davis, M. (1992). The Role of the Amygdala in Fear and Anxiety. Annual Review of Neuroscience, 15(1), 353-375. https://doi.org/10.1146/annurev.ne.15.030192.002033

Derakshan, N., \& Koster, E. H. W. (2010). Processing efficiency in anxiety: Evidence from eyemovements during visual search. Behavior Research and Therapy, 1180-1185. https://doi.org/10.1016/j.brat.2010.08.009

Dolcos, F., LaBar, K. S., \& Cabeza, R. (2004). Interaction between the amygdala and the medial temporal lobe memory system predicts better memory for emotional events. Neuron, 42(5), 855-863. https://doi.org/10.1016/So896-6273(04)00289-2

Kaplan, R. L., Van Damme, I., Levine, L. J., \& Loftus, E. F. (2016). Emotion and False Memory. 
Emotion Review, 8(1), 8-13. https://doi.org/10.1177/1754073915601228

Kassin, S. M., Ellsworth, P. C., \& Smith, V. L. (1989). The "General Acceptance" of Psychological Research on Eyewitness Testimony: A Survey of the Experts. American Psychologist, 44(8), 1089-1098. https://doi.org/10.1037/0003-066X.44.8.1089

LeDoux, J. (2007, October 23). The amygdala. Current Biology, Vol. 17. https://doi.org/10.1016/j.cub.2007.08.005

Loftus, E. F., \& Pickrell, J. E. (1995). The Formation of False Memories. Psychiatric Annals, 25(12), 720-725. https://doi.org/10.3928/0048-5713-19951201-07

Madan, C. R., Caplan, J. B., Lau, C. S. M., \& Fujiwara, E. (2012). Emotional arousal does not enhance association-memory. Journal of Memory and Language, 66(4), 695-716. https://doi.org/10.1016/j.jml.2012.04.001

Mather, M., \& Knight, M. (2008). The Emotional Harbinger Effect: Poor Context Memory for Cues That Previously Predicted Something Arousing. Emotion, 8(6), 850-860. https://doi.org/10.1037/a0014087

Montaldi, D., \& Mayes, A. R. (2010). The role of recollection and familiarity in the functional differentiation of the medial temporal lobes. Hippocampus, 2O(11), 1291-1314. https://doi.org/10.1002/hipo.20853

O’Keefe, J., \& Nadel, L. (1978). The Hippocampus as a Cognitive Map. New York: Clarendon Press.

Öhman, A., Flykt, A., \& Esteves, F. (2001). Emotion drives attention: Detecting the snake in the grass. Journal of Experimental Psychology: General, 130(3), 466-478. https://doi.org/10.1037/oo96-3445.130.3.466

Pezdek, K. (2003). Event memory and autobiographical memory for the events of September 11, 2001. Applied Cognitive Psychology, 17(9), 1033-1045. https://doi.org/10.1002/acp.984

Phelps, E. A. (2004, April). Human emotion and memory: Interactions of the amygdala and hippocampal complex. Current Opinion in Neurobiology, Vol. 14, pp. 198-202. https://doi.org/10.1016/j.conb.2004.03.015

Power, M., Philippot, P., \& Hess, U. (2010). Emotion-Focused Cognitive Therapy. In EmotionFocused Cognitive Therapy. https://doi.org/10.1002/9780470660065

Rimmele, U., Davachi, L., Petrov, R., Dougal, S., \& Phelps, E. A. (2011). Emotion Enhances the Subjective Feeling of Remembering, Despite Lower Accuracy for Contextual Details. Emotion, 11(3), 553-562. https://doi.org/10.1037/a0024246

Schupp, H. T., Junghöfer, M., Weike, A. I., \& Hamm, A. O. (2003). Emotional facilitation of sensory processing in the visual cortex. Psychological Science, 14(1), 7-13. 
https://doi.org/10.1111/1467-9280.01411

Stark, C. E. L., Bayley, P. J., \& Squire, L. R. (2002). Recognition memory for single items and for associations is similarly impaired following damage to the hippocampal region. Learning and Memory, 9(5), 238-242. https://doi.org/10.1101/lm.51802

Tottenham, N., Tanaka, J. W., Leon, A. C., McCarry, T., Nurse, M., Hare, T. A., ... Nelson, C. (2009). The NimStim set of facial expressions: Judgments from untrained research participants. Psychiatry Research, 168(3), 242-249.

https://doi.org/10.1016/j.psychres.2008.05.006

Touryan, S. R., Marian, D. E., \& Shimamura, A. P. (2007). Effect of negative emotional pictures on associative memory for peripheral information. Memory, 15(2), 154-166. https://doi.org/10.1080/o9658210601151310

Van Der Kolk, B. A. (1998). Trauma and memory. Psychiatry and Clinical Neurosciences, 52(S1), S57-S69. https://doi.org/10.1046/j.1440-1819.1998.0520s5s97.x 